\title{
Sestrin2 inhibits YAP activation and negatively regulates corneal epithelial cell proliferation
}

\author{
Ji-Su Lee ${ }^{1,2}$, Hwan-Woo Park ${ }^{3}$ Kyong Jin Cho ${ }^{4}$ and Jungmook Lyu ${ }^{1,2}$
}

\begin{abstract}
Corneal wound healing is essential for the maintenance of corneal integrity and transparency and involves a series of physiological processes that depend on the proliferation of epithelial cells. However, the molecular mechanisms that control corneal epithelial cell proliferation are poorly understood. Here, we show that Sestrin2, a stress-inducible protein, is downregulated in the corneal epithelium during wound healing and that the proliferation of epithelial basal cells is enhanced in Sestrin2-deficient mice. We also show that YAP, a major downstream effector of the Hippo signaling pathway, regulates cell proliferation during corneal epithelial wound repair and that Sestrin2 suppresses its activity. Moreover, increased levels of reactive oxygen species in the Sestrin2-deficient corneal epithelium promote the nuclear localization and dephosphorylation of YAP, activating it to enhance the proliferation of corneal epithelial cells. These results reveal that Sestrin2 is a negative regulator of YAP, which regulates the proliferative capacity of basal epithelial cells, and may serve as a potential therapeutic target for corneal epithelial damage.
\end{abstract}

\section{Introduction}

The corneal epithelium is the outermost layer of the cornea. It protects the eye from environmental injury and is required to maintain corneal transparency. The corneal epithelium is composed of several layers of superficial squamous cells, multiple layers of wing cells, and a single layer of basal cells, all of which are regenerated throughout life by corneal epithelial stem cells (CESCs) ${ }^{1}$. The repair process of corneal epithelial wound healing, caused by various injuries, involves three cellular events: basal cell proliferation, cell migration from the surrounding epithelium to the wound site, and cell differentiation into stratified layers ${ }^{1-3}$. These cellular events depend on complex interactions among extrinsic and intrinsic signaling pathways.

Sestrins (Sesns) are a family of highly conserved, stress-inducible proteins. Sesn1, Sesn2, and Sesn3 are

\footnotetext{
Correspondence: Jungmook Lyu (lyujm@konyang.ac.kr)

${ }^{1}$ Myung-Gok Eye Research Institute, Konyang University, 158 Gwanjeodong-ro, Seo-gu, Daejeon 35365, South Korea

2Department of Medical Science, Konyang University, 158 Gwanjeodong-ro, Seo-gu, Daejeon 35365, South Korea
}

Full list of author information is available at the end of the article found in mammals. Sesn2 has been most extensively studied as a stress response gene ${ }^{4}$. It is known to suppress reactive oxygen species (ROS) production through its antioxidant activity and provides cytoprotection from a variety of noxious stimuli, such as that elicited by oxidative stress, genotoxic stress, endoplasmic reticulum (ER) stress, and hypoxia ${ }^{5}$. Recent studies have shown that Sesn2 negatively regulates cell proliferation by inhibiting mammalian target of rapamycin complex 1 (mTORC1) signaling via the activation of AMP-activated protein kinase (AMPK) $)^{6,7}$. mTOR, a serine/threonine kinase, regulates key cellular functions, including growth, proliferation, and metabolism ${ }^{8,9}$. mTOR kinase exists as two large complexes, mTORC1 and mTORC2. mTORC1 phosphorylates and activates S6K, which in turn phosphorylates ribosomal protein $\mathrm{S}^{10,11}$. In the cornea, mTOR signaling is known to be involved in scarring, neovascularization, and inflammation ${ }^{12-15}$. However, it is unclear whether Sesn2 regulates the proliferation of corneal epithelial cells.

The Hippo signaling pathway is involved in the regulation of organ size, tissue regeneration, and stem cell self-renewal $^{16-18}$. In mammals, the Hippo pathway is 
composed of a core kinase cascade consisting of mammalian Ste20-like kinase 1/2 (MST1/2) and large tumor suppressor 1/2 (LATS1/2) and transcriptional coactivators. Two key downstream transcriptional coactivators, Yes-associated protein (YAP) and transcriptional coactivator with PDZ-binding motif (TAZ), induce the expression of genes that are involved in cell proliferation, survival, and migration ${ }^{19-21}$. When the Hippo signaling pathway is activated by upstream signals, MST1/2 are phosphorylated and activate LATS1/2. Activated LATS1/ 2 phosphorylate the transcription coactivator YAP at Ser127, which accumulates in the cytoplasm and is thus excluded from the nucleus ${ }^{22-26}$. Inactivation of the Hippo signaling pathway induces the dephosphorylation of YAP and its translocation into the nucleus to promote TEAD binding and the activation of gene transcription ${ }^{25}$. Recent studies have revealed that YAP is expressed in corneal basal epithelial cells and plays an essential role in maintaining their proliferation ${ }^{27}$. However, the underlying mechanism of YAP regulation in corneal wound healing remains unknown.

In this study, we show that Sesn2 deficiency promotes the proliferation of corneal epithelial cells during corneal wound healing in vitro and in vivo. We also show that YAP is activated in Sesn2-deficient corneal epithelial cells, which results in an increase in ROS levels. These results demonstrate that Sesn2 suppresses YAP activity and negatively regulates corneal epithelial cell proliferation.

\section{Materials and methods}

\section{Cell culture and lentiviral infection}

Human corneal epithelial (hCET) cells were provided by Kaoru Araki-Sasaki (Osaka University, Osaka, Japan). The cells were cultured at $37^{\circ} \mathrm{C}$ in Dulbecco's modified Eagle's medium (DMEM)/F-12 (1:1; Corning, NY, USA) supplemented with $5 \%$ fetal bovine serum (Corning), $1 \times$ penicillin/streptomycin (WELGENE, Gyeongsan, Korea), $500 \mathrm{ng} / \mathrm{ml}$ hydrocortisone (Sigma-Aldrich, St Louis, MO, USA) and $30 \mathrm{ng} / \mathrm{ml}$ cholera toxin (Sigma-Aldrich). For Sesn2- and/or YAP-knockdown hCET cells, lentiviruses expressing Sesn 2 shRNA, YAP shRNA, or wild-type Sesn2 were generated as previously described ${ }^{5}$ and transfected into the hCET cells. The Sesn 2 shRNA and wild-type Sesn2 lentiviral plasmids were kindly provided by Andrei V. Budanov (Trinity College, Dublin, Ireland), and the YAP shRNA lentiviral plasmid (\#42540) was obtained from Addgene (Cambridge, MA, USA).

\section{In vivo and in vitro wound healing assays}

Sesn $2^{-1-}$ mice with a CL57BL/6 background were kindly provided by Dr. Seo Goo Rhee (Yonsei University, South Korea). Eight-week-old Sesn $2^{-/-}$and Sesn $2^{+/+}$ mice were administered general anesthesia with Zoletil
(Virbac, Carros, France) and Rompun (Bayer, Seoul, South Korea). A circular defect was produced in the central corneal epithelium using a sterile disposable biopsy punch with a diameter of 2 or $2.5 \mathrm{~mm}$ (Kai, Tokyo, Japan). The corneal epithelial defects were stained with fluorescein and photographed at 0 and $24 \mathrm{~h}$ for the $2 \mathrm{~mm}$ defect and at $0,24,48,72$, and $96 \mathrm{~h}$ for the $2.5 \mathrm{~mm}$ defect.

For the in vitro wound healing assay, Ibidi culture inserts (Ibidi GmbH, Münich, Germany) were placed at the bottom of the wells in a 6-well plate. hCET cells were seeded into each well and incubated for $24 \mathrm{~h}$. Thereafter, the Ibidi culture inserts were removed to create a wound. The cells were photographed at 0,12 , and $24 \mathrm{~h}$. Wound healing was measured by determining the percentage of the wound area.

\section{Immunostaining and western blotting}

For immunostaining, cells grown on coverslips were fixed in $4 \%$ paraformaldehyde and permeabilized with $0.1 \%$ Triton X-100. The fixed cells and corneal sections were incubated with a blocking solution containing $1 \%$ bovine serum albumin (BSA) and 5\% normal goat serum for $1 \mathrm{~h}$ and incubated overnight at $4{ }^{\circ} \mathrm{C}$ with anti-pS6 and anti-Yap antibodies. Thereafter, the cells were rinsed with PBS, incubated with secondary antibodies (Alexa Fluor 488) at room temperature for $1 \mathrm{~h}$, and counterstained with Hoechst 33342. Images were acquired using a fluorescence microscope with an AxioCam camera (Zeiss, Jena, Germany).

For western blotting, proteins were extracted in lysis buffer (25 mM Tris- $\mathrm{HCl}$ (pH 7.4), $150 \mathrm{mM}$ sodium chloride, $5 \mathrm{mM}$ EDTA, $1 \times$ Triton X-100, 10\% glycerol, $10 \mathrm{mM}$ sodium pyrophosphate, $10 \mathrm{mM} \beta$-glycerophosphate, $1 \mathrm{mM}$ sodium orthovanadate, $1 \times$ protease inhibitor, $10 \mathrm{mM}$ sodium fluoride, and $1 \mathrm{mM}$ phenylmethylsulfonyl fluoride). The concentration of proteins in the total cell extracts was determined using a bicinchoninic acid assay (Thermo Fisher Scientific, Waltham, MA, USA). Thereafter, equal amounts of proteins were separated by $10 \%$ SDS-PAGE and transferred to polyvinylidene difluoride membranes, which were then blocked with $5 \%$ nonfat dry milk or $5 \%$ BSA in TBS with $0.02 \%$ Tween- 20 for $1 \mathrm{~h}$. The membranes were incubated overnight with primary antibodies at their optimal concentration at $4{ }^{\circ} \mathrm{C}$. The next day, the membranes were washed with TBST three times for 5 min each time and incubated with horseradish peroxidaseconjugated secondary antibody for $1.5 \mathrm{~h}$ at room temperature. The immunoreactive bands were visualized using an enhanced chemiluminescence detection kit (Bio-Rad, Hercules, CA, USA). The following antibodies were used: phospho-YAP (Cell Signaling, Cat. 4911), YAP (Cell Signaling, Cat. 14074), Sestrin2 (provided by Jun Hee Lee, University of Michigan), phospho-S6 (Cell Signaling, Cat. 2211), and S6 (Santa Cruz, Cat. 74459). 


\section{BrdU and EdU incorporation assays}

For in vivo proliferation analysis, Sesn $2^{+/+}$and Sesn $2^{-/-}$ mice were injured and injected intraperitoneally with 5-bromo-2'-deoxyuridine (BrdU) $(100 \mathrm{mg} / \mathrm{kg})$. The mouse eyes were collected $48 \mathrm{~h}$ after injection and fixed. Corneal sections were incubated in $1 \mathrm{M} \mathrm{HCl}$ for $30 \mathrm{~min}$ at $37^{\circ} \mathrm{C}$ and washed in $0.5 \mathrm{mM}$ borate buffer before incubation with an anti-BrdU antibody. Cell proliferation was assessed by immunostaining BrdU (Abcam, Cambridge, UK). Nuclei were stained with Hoechst 33342.

For in vitro proliferation analysis, hCET cells were grown on coverslips in culture medium, and $1 \mu \mathrm{M} \mathrm{5-}$ ethyl-2'-deoxyuridine (EdU) was added. After incubation with EdU for $6 \mathrm{~h}$, + cells were washed three times with PBS, fixed in 4\% PFA, and incubated in $200 \mu \mathrm{l}$ of Click-iT reaction mix (Click-iT EdU Alexa Fluor 488 Imaging Kit; Invitrogen, Carlsbad, CA, USA) for $30 \mathrm{~min}$. Cell proliferation was measured by determining the amount of EdU incorporation in each cell.

\section{Luciferase assay}

For the luciferase assay, hCET cells expressing Sesn 2 shRNA or control shRNA were seeded in 24-well plates. Cells were transfected with the YAP reporter 8xGTIIClux (Addgene, Cambridge, USA) and an internal control, pRL-TK. The cells were harvested $24 \mathrm{~h}$ after transfection and analyzed using a dual-luciferase reporter assay kit (Promega, Wisconsin, USA).

\section{ROS detection}

Oxidation-sensitive fluorescent dye dihydroethidium (DHE) was used to assess intracellular ROS levels. Injured corneal sections from Sesn $2^{+/+}$and Sesn $2^{-/-}$mice were washed in PBS and incubated in $10 \mu \mathrm{M}$ DHE for $30 \mathrm{~min}$. Then, the corneal sections were washed in PBS and mounted onto glass slides before images were acquired.

\section{Cell cycle assay}

Cell cycle analyses were performed using fluorescenceactivated cell sorting (FACS). hCET cells expressing control shRNA or Sesn 2 shRNA were harvested from a 6well plate and fixed overnight in $70 \%$ ethanol at $20^{\circ} \mathrm{C}$. After centrifugation at $800 \mathrm{rcf}$ for $3 \mathrm{~min}$, the pellet was resuspended in PBS and stained with a cell cycle solution (Tali® Cell Cycle kit; Invitrogen, Carlsbad, CA, USA) for 30 min under dark conditions. The cell cycle profile was analyzed using a flow cytometer (NovoCyte, ACEA Biosciences, San Diego, CA, USA).

\section{Quantitation of nuclear YAP}

To determine whether YAP translocated into the nucleus of the corneal epithelial cells in the Sesn $2^{-1-}$ mice treated with NAC or DMSO, mouse corneal sections were immunostained with an anti-YAP antibody. The values obtained from at least two experiments were averaged and are presented as the means \pm standard deviation (SD). Hoechst 33258 was used for nuclear staining. Fluorescence images were acquired using a fluorescence microscope with an AxioCam camera (Zeiss, Jena, Germany). For each nucleus, the fluorescence intensity of the antiYAP secondary antibody was measured using ZEN software (Zeiss). A minimum of 45 corneal epithelial cells were examined.

\section{Results}

\section{Sesn2 negatively regulates corneal epithelial cell proliferation}

To investigate the function of Sesn2 in corneal wound healing, corneas of Sesn $2^{+/+}$and Sesn $2^{-/-}$mice were first denuded of their epithelium, and corneal wound healing was monitored by fluorescein staining for 4 days. The corneal wound area in Sesn $2^{-1-}$ mice was almost completely healed within $96 \mathrm{~h}$, whereas that of Sesn $2^{+/+}$mice remained unhealed (Fig. 1a). The percentage of wound area was determined in Sesn $2^{-/-}$and $\operatorname{Sesn} 2^{+/+}$mice. The results revealed that $\operatorname{Sesn} 2^{-1-}$ mice had a significantly faster rate of wound closure than Sesn $2^{+/+}$mice (Fig. 1b). Western blot analysis of corneal epithelial lysates revealed that Sesn2 protein expression was decreased in the injured cornea compared to that in the normal cornea of $\operatorname{Sesn} 2^{+/+}$mice (Fig. 1c). These results suggest that Sesn2 can inhibit corneal epithelial wound healing. To address this possibility, hCET cells transduced with lentiviruses expressing Sesn 2 shRNA or control shRNA were seeded into wound assay chambers and monitored for $24 \mathrm{~h}$ after wounding. At 12 and $24 \mathrm{~h}$, the wound closure rate of hCET cells expressing Sesn 2 shRNA was significantly higher than that of those expressing control shRNA (Fig. 1d, e). In addition, when wild-type Sesn 2 was reexpressed in Sesn2-deficient hCET cells, wound closure was delayed (Supplementary Fig. S1). Taken together, these results suggest that Sesn2 deficiency enhances corneal epithelial wound healing.

To determine whether Sesn 2 regulates the proliferative capacity of epithelial cells during corneal wound healing, we injected Sesn $2^{+/+}$and Sesn $2^{-/-}$mice with bromodeoxyuridine (BrdU) and mechanically induced corneal epithelial wounds. BrdU-incorporated cells were identified by immunostaining using an anti-BrdU antibody $48 \mathrm{~h}$ of injury. BrdU-positive cells were detected in both the central and peripheral regions of the corneal epithelium (Fig. 2a). Moreover, the number of BrdU-positive cells in the cornea of Sesn $2^{-l-}$ mice was significantly higher than that in the cornea of Sesn $2^{+/+}$mice (Fig. 2a, b). In addition, the number of EdU-positive cells was significantly increased in cultures expressing Sesn2 shRNA compared to cultures expressing control shRNA (Fig. 2c, d). To further confirm the effect of Sesn2 on the proliferative 

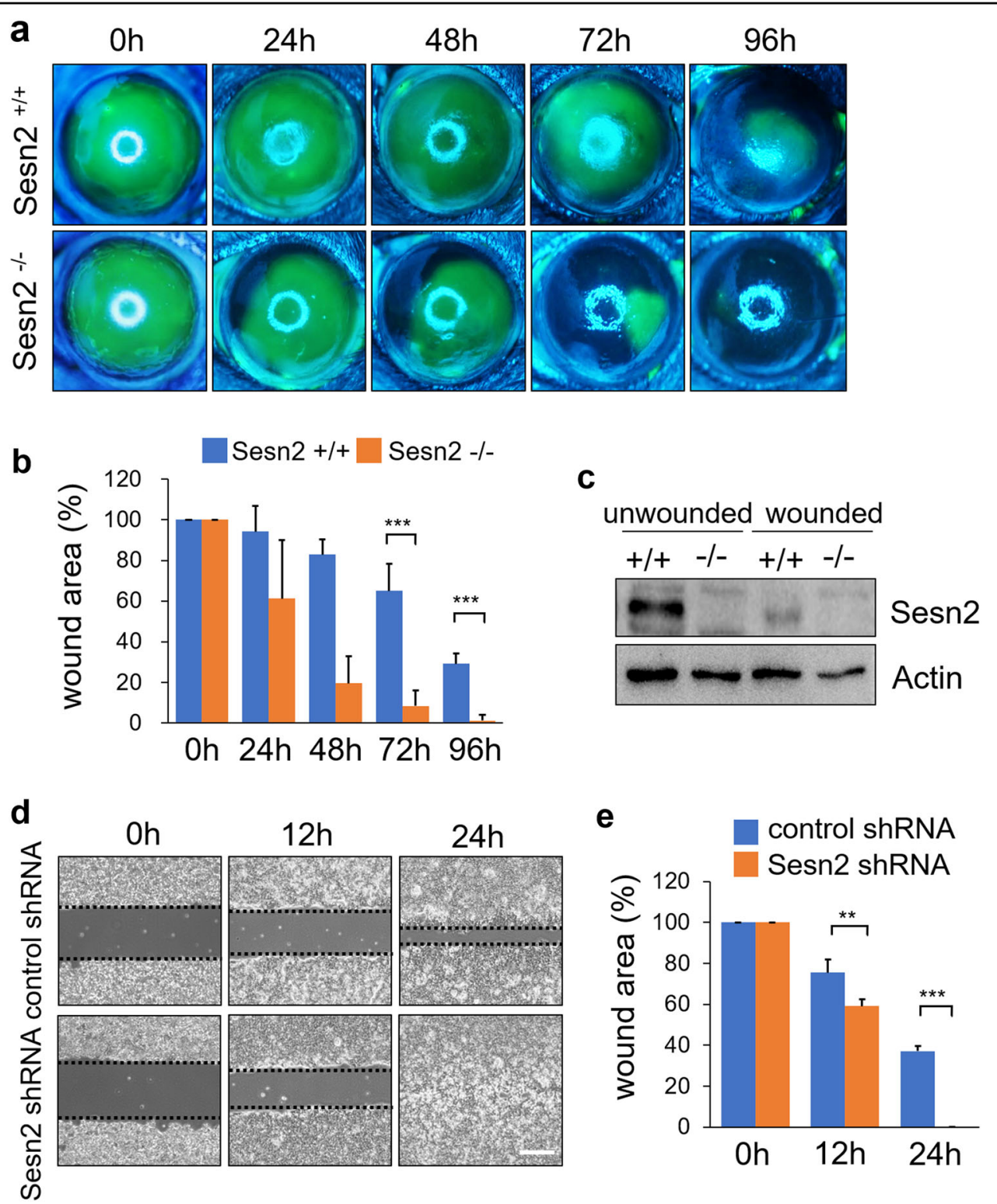

Fig. 1 Sesn2 deficiency enhances corneal wound healing. a Representative photographs of the fluorescein-stained corneas of Sesn $2^{-/-}$and Sesn $2^{+/+}$mice at $0,24,48,72$, and $96 \mathrm{~h}$ after injury using a $2.5 \mathrm{~mm}$ punch. The fluorescein-stained area represents the corneal epithelial wound area. By $96 \mathrm{~h}$, the corneal wound area in Sesn $2^{-1-}$ mice was healed, whereas that of Sesn $2^{+/+}$mice remained open. $\mathbf{b}$ Quantitative analysis of the corneal wound area of Sesn $2^{-/-}$and $+/+$mice. A total of 12 eyes from Sesn $2^{-/-}$and Sesn $2^{+/+}$mice were used to measure the epithelial wound area size. The wound area was significantly smaller in Sesn $2^{-/-}$mice than in Sesn $2^{+/+}$mice. c Corneal tissues from Sesn2 $2^{-/-}$and Sesn $2^{+/+}$mice were lysed $48 \mathrm{~h}$ after injury and subjected to western blotting with Sesn2 and actin antibodies. $\mathbf{d}$ In vitro wound healing assays of hCET cells expressing Sesn2 shRNA and control shRNA. hCET cells expressing Sesn2 shRNA or control shRNA were seeded on both sides of a wound chamber and allowed to attach for $12 \mathrm{~h}$. The chamber was removed, and the wound areas were photographed immediately at 0, 12, and 24 h. Dotted lines indicate wound borders at the beginning of the assay. e Quantitative analysis of the wound areas of hCET cells expressing Sesn2 shRNA and control shRNA at 0, 12, and $24 \mathrm{~h}$. The rate of wound closure in hCET cells expressing Sesn2 shRNA was significantly higher than in hCET cells expressing control shRNA. Error bars represent the means \pm SD of three independent experiments. Two-tailed Student's $t$-test $\left(^{* *} p<0.01\right.$, ${ }^{* * *} p<$ 0.001). Scale bar, $300 \mu \mathrm{m}$.

potential of hCET cells, the distribution of hCET cells expressing control shRNA or Sesn2 shRNA in different phases of the cell cycle was analyzed. The proportion of Sesn 2 shRNA-expressing hCET cells in the S/G2 phase was higher than that of control shRNA-expressing hCET cells (Fig. 2e). These results suggest that Sesn2 deficiency can facilitate the proliferation of corneal epithelial cells by regulating the S/G2 phase of the cell cycle. 

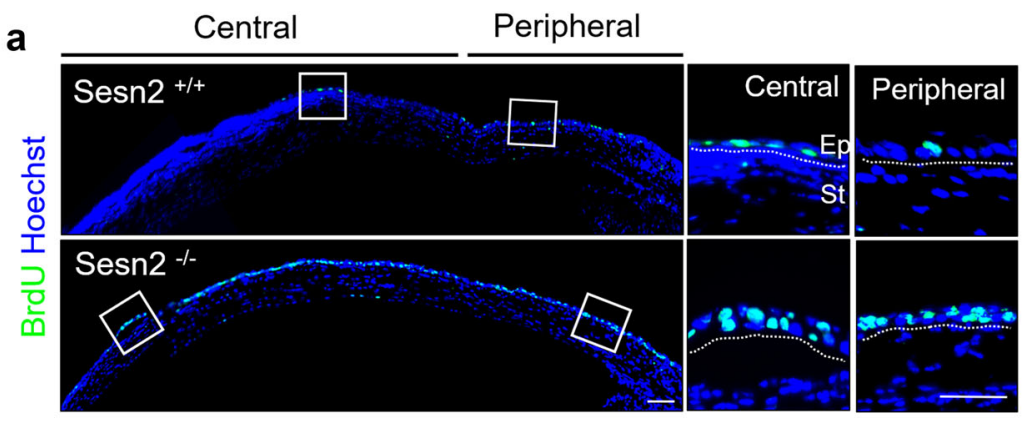

\section{b}
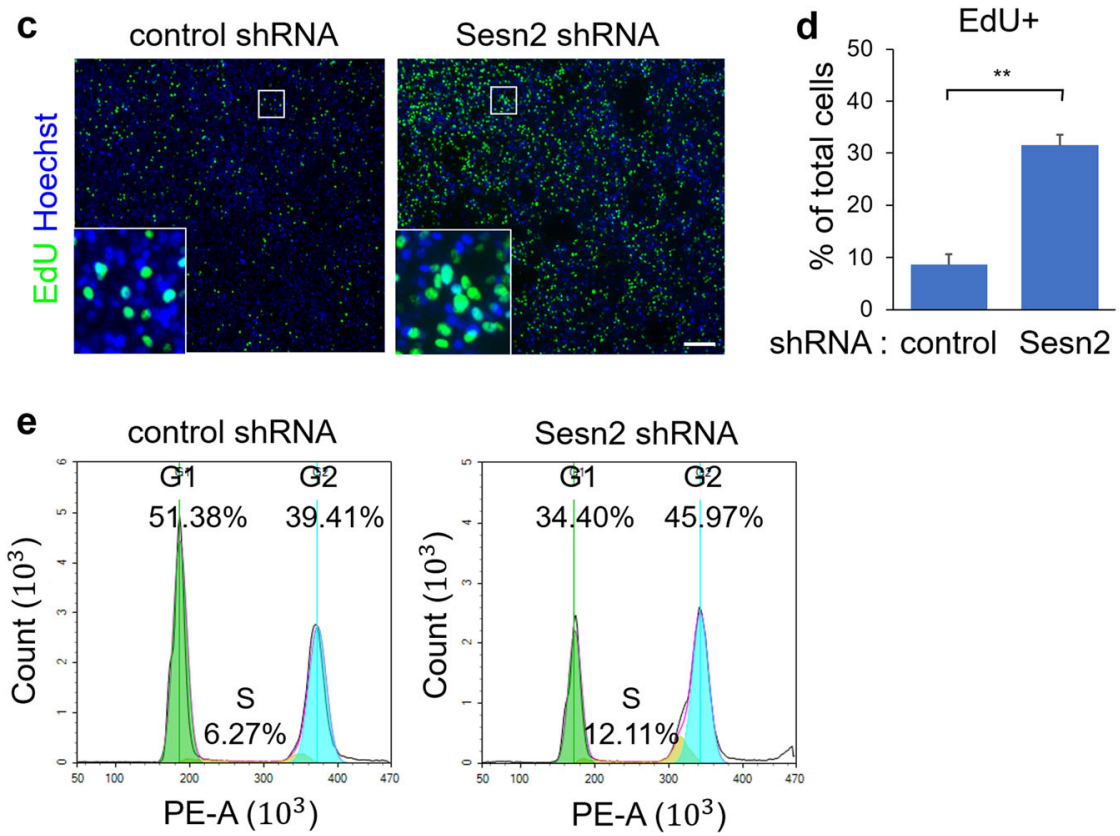

Fig. 2 Sesn 2 deficiency promotes corneal epithelial cell proliferation. a BrdU was injected into Sesn $2^{-/-}$and Sesn $2^{+/+}$mice after injury, and the mice were sacrificed $48 \mathrm{~h}$. Corneal sections were stained using an anti-BrdU antibody (green). Hoechst dye was used as a counterstain (blue). BrdUpositive cells were observed in the central and peripheral regions of corneal epithelial layers and were primarily detected in Sesn $2^{-/-}$mice. Dotted lines indicate the basement membrane. $\mathbf{b}$ Quantitative analysis of BrdU-positive cells in the corneal epithelium of Sesn2 $2^{-/-}$and Sesn $2^{+/+}$mice after injury. c EdU incorporation assay of hCET cells expressing Sesn2 shRNA or control shRNA. Cells were incubated with $10 \mu \mathrm{M}$ EdU for $4 \mathrm{~h}$. d Percentage of EdU-positive cells. The number of EdU-positive Sesn2-deficient hCET cells was significantly increased. e Distribution of cells in different cell cycle phases. The proportion of Sesn2-deficient hCET cells in the S and G2 phases of the cell cycle was higher than that of control cells. Error bars represent the means \pm SD of three independent experiments. Two-tailed Student's t-test ( $\left.{ }^{* *} p<0.01\right)$. Scale bars, $50 \mu \mathrm{m}$ in (a); $100 \mu \mathrm{m}$ in (c).

\section{Sesn2 deficiency increases mTOR signaling activity}

We next sought to determine whether Sesn2 regulates mTOR signaling activity in corneal epithelial wound healing. The sections of injured corneas from Sesn $2^{+/+}$ and $\operatorname{Ses} n 2^{-1-}$ mice were immunostained with an antibody against the phosphorylated S6 ribosomal protein, a marker of mTORC1 activity. Phospho-S6 proteins were detected in the basal cells of the corneal epithelium (Fig. $3 a)$. The expression of phospho-S6 protein in the central and peripheral corneal regions of $\operatorname{Ses} n 2^{-1-}$ mice was higher than that in these regions of the Sesn $2^{+/+}$mice. Moreover, western blotting revealed that pS6 expression was increased in the hCET cells expressing Sesn 2 shRNA compared to that of the cells expressing control shRNA
(Fig. 3b). To evaluate whether mTOR signaling promotes wound healing in Sesn2-deficient corneas, the corneal epithelium of Sesn $2^{-1-}$ mice was mechanically denuded and treated with rapamycin, an inhibitor of mTORC1, or DMSO. Wound closure was delayed in the rapamycintreated Sesn $2^{-1-}$ mice, whereas the wound area was completely healed in DMSO-treated Sesn $2^{-1-}$ mice $24 \mathrm{~h}$ after injury (Fig. 3c). The wound area percentage was quantitated; the rate of corneal epithelial wound closure in rapamycin-treated Sesn $2^{-1-}$ mice was significantly delayed compared to that in DMSO-treated counterparts (Fig. 3d). We also observed delayed wound closure in rapamycin-treated Sesn $2^{+/+}$mice (Supplementary Fig. $\mathrm{S} 2 \mathrm{a}, \mathrm{b})$, although the percentage of reduction in the 


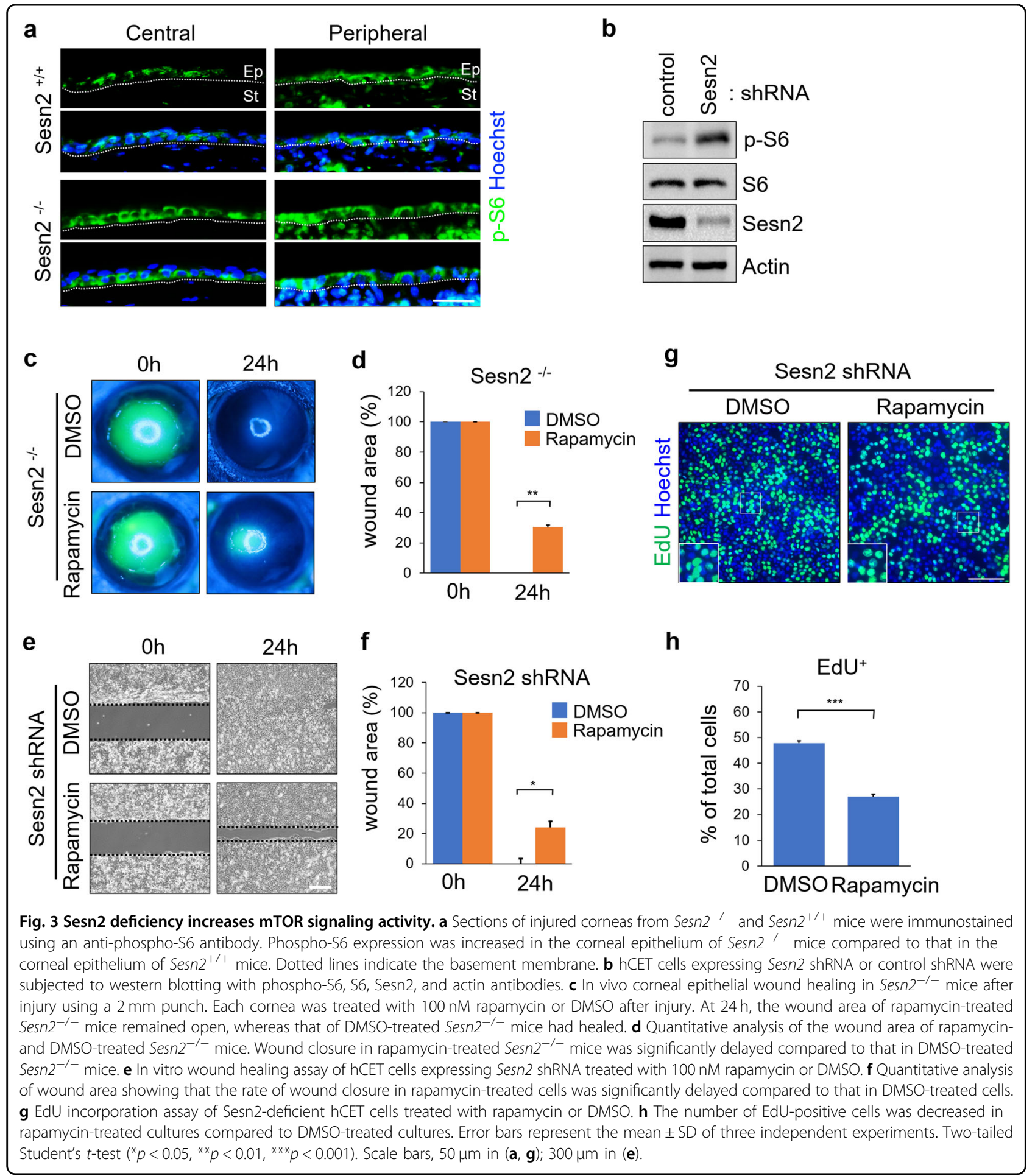

wound closure rate was markedly higher in $\operatorname{Sesn} 2^{-1-}$ mice than in Sesn $2^{+/+}$mice. In addition, the in vitro wound assay using Sesn2-deficient hCET cells treated with rapamycin or DMSO produced results similar to those found for the mice (Fig. 3e, f). However, no significant difference in the percentage of wound area was observed between the control shRNA-expressing hCET cells treated with rapamycin and DMSO (Supplementary Fig. S2c, d). This finding indicates that rapamycin affects corneal epithelial wound healing in a Sesn2-dependent manner. To test the effect of rapamycin on the proliferation of corneal epithelial cells, we treated hCET cells expressing 
Sesn2 shRNA with rapamycin and DMSO and performed an EdU incorporation assay. Rapamycin treatment significantly decreased the number of EdU-positive cells (Fig. $3 \mathrm{~g}, \mathrm{~h}$ ). Taken together, these results demonstrate that Sesn2 deficiency activates mTOR signaling and promotes the proliferation of corneal epithelial cells. Therefore, mTOR signaling promotes corneal wound healing and is negatively regulated by Sesn2.

\section{Sesn2 negatively regulates YAP activity}

Sesn2 can potentially regulate the Hippo signaling pathway because AMPK and mTORC1 are known to regulate YAP activity. To investigate whether the loss of Sesn2 affects YAP activity in corneal epithelial cells, we analyzed the expression of YAP protein in normal and injured corneas of Sesn $2^{+/+}$and Sesn $2^{-/-}$mice. Immunostaining with an anti-YAP antibody revealed that YAP protein is expressed in the basal layer of the corneal epithelium (Fig. 4a). In the normal corneas, YAP protein was mainly detected in the cytoplasm of basal epithelial cells in the central region, whereas its protein was expressed in both the cytoplasm and nucleus of epithelial cells in the peripheral region. Interestingly, the injured corneas of Sesn $2^{+/+}$mice exhibited increased nuclear localization of YAP protein in the basal epithelial cells in the central region (Fig. 4a, arrows) but not in the epithelial cells in the peripheral region. The number of basal epithelial cells exhibiting nuclear localization of YAP in the central region was increased in the injured cornea of $\operatorname{Sesn} 2^{-1-}$ mice. Fluorescence intensity analysis corroborated this finding by showing that nuclear YAP was detected more frequently in the corneal epithelial cells of $\operatorname{Ses} n 2^{-1-}$ mice than in the epithelial cells of Sesn $2^{+/+}$mice (Fig. 4b). In addition, the number of cells showing predominant nuclear localization of YAP in cultures expressing Sesn2 shRNA was higher than in those expressing control shRNA (Fig. 4c). YAP protein is dephosphorylated at the serine 127 residue upon translocation into the nucleus and is subsequently activated. To determine whether Sesn2 negatively regulates YAP activation, hCET cells transduced with lentiviruses expressing Sesn2 shRNA or control shRNA were subjected to western blotting using an anti-phospho-YAP (serine 127) antibody. As expected, the expression of phosphorylated YAP protein in cultures expressing Sesn 2 shRNA was lower than that in cultures expressing control shRNA (Fig. 4d). In addition, re-expression of wild-type Sesn2 in Sesn2-deficient hCET cells resulted in an increase in the level of phosphorylated YAP protein (Supplementary Fig. S3a). Furthermore, the GTIIC reporter activity of YAP was increased in the cultured cells expressing Sesn2 shRNA compared to those expressing control shRNA (Fig. 4e). These results demonstrate that Sesn2 deficiency increases YAP activity.
To further investigate whether YAP is required for Sesn2 deficiency-mediated corneal wound healing, the mechanically denuded corneal epithelium in Sesn $2^{-1-}$ mice was treated with verteporfin, a porphyrin compound that blocks the interaction between YAP and TEAD and represses YAP function ${ }^{28}$, or DMSO. The rate of corneal epithelial wound closure was significantly delayed in verteporfin-treated corneas compared to DMSO-treated corneas (Fig. 4f, Supplementary Fig. S3b). We performed an in vitro wound assay using Sesn2-deficient hCET cells transduced with lentiviruses expressing YAP shRNA or control shRNA and observed delayed wound closure in cultures expressing YAP shRNA (Supplementary Fig. S3c). The wound area was completely healed in cultures expressing control shRNA after $24 \mathrm{~h}$. The percentage of the wound area was quantitated, and the results showed that YAP deficiency decreased the rate of wound closure (Fig. 4g). Next, we tested whether YAP promotes the proliferation of corneal epithelial cells. The number of EdUpositive cells in Sesn2-deficient hCET cells expressing YAP shRNA was significantly decreased as compared with that in Sesn2-deficient hCET cells expressing control shRNA (Fig. 4h, i). These results indicate that YAP is required to regulate the proliferation of hCET cells in Sesn2 deficiency-mediated corneal wound healing.

To determine whether AMPK regulates YAP activity, we treated hCET cells expressing Sesn 2 shRNA or control shRNA with 5-aminoimidazole-4carboxamideribonucleotide (AICAR), an AMPK activator, or DMSO. Western blot analysis showed that the expression of phospho-S6 protein was decreased in AICAR-treated cultured cells expressing Sesn 2 shRNA compared to DMSO-treated cultures expressing Sesn2 shRNA (Fig. 4j). However, there was no difference in the expression of phospho-YAP protein between AICARtreated and DMSO-treated hCET cells expressing Sesn2 shRNA. Moreover, rapamycin treatment did not affect YAP activity in hCET cells expressing Sesn 2 shRNA, while the expression of phospho-S6 protein was decreased in these cells (Fig. 4k). These results suggest that Sesn2 regulates YAP activation via an AMPK- and mTORC1independent mechanism.

\section{Sesn2 deficiency activates YAP by regulating ROS production}

It has been previously reported that Sesn2 suppresses ROS production. To determine whether ROS production is increased in Sesn2-deficient corneas, we stained cells with DHE to specifically detect ROS levels in the injured corneas of Sesn $2^{+/+}$and Sesn $2^{-/-}$mice. The fluorescence intensity for DHE in the corneal epithelial cells of Sesn $2^{-1-}$ mice was higher than that of the corneal epithelial cells in $\operatorname{Sesn} 2^{+/+}$mice, indicating increased levels of ROS in 


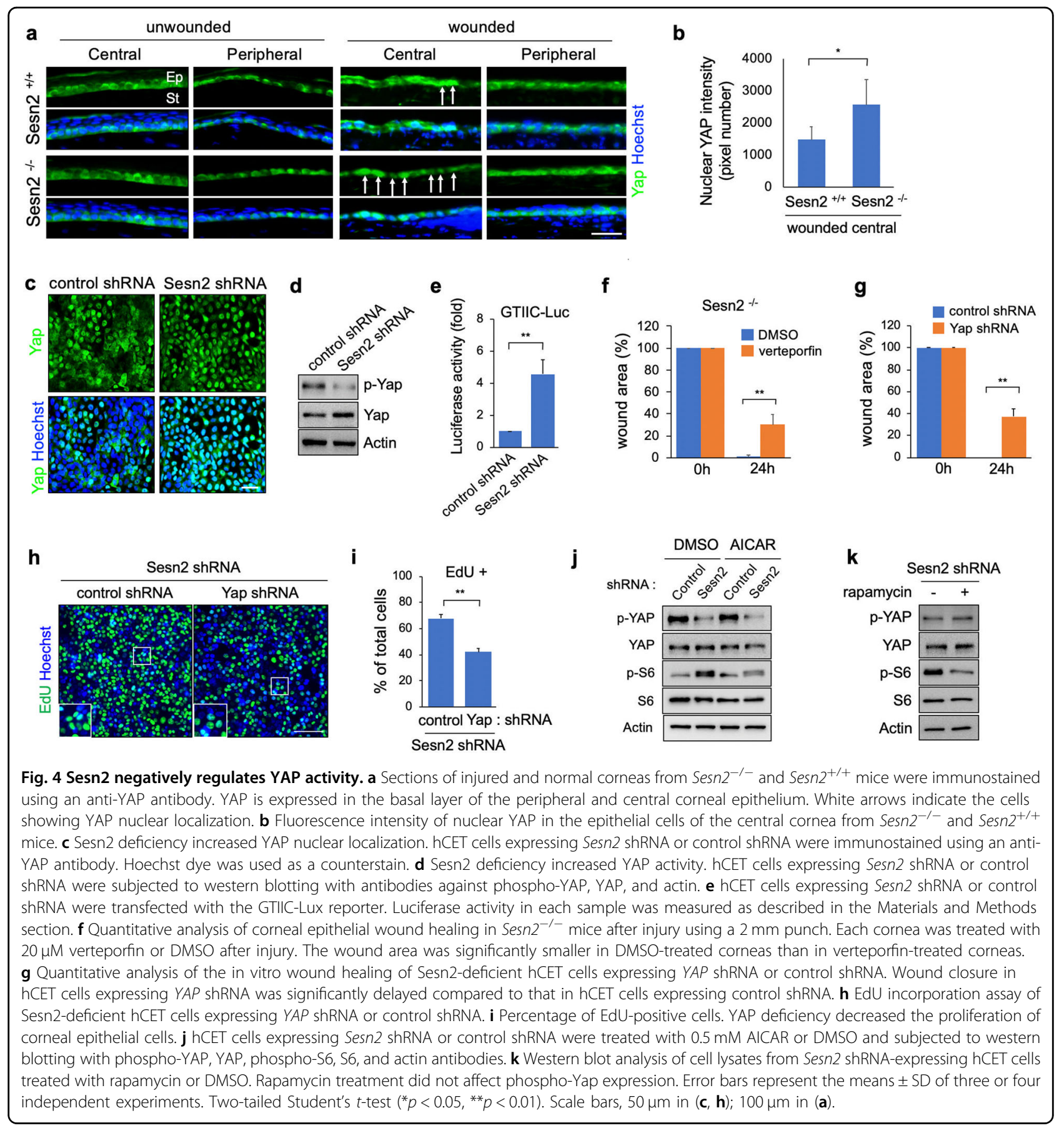

Sesn2-deficient corneas (Fig. 5a). We next treated the injured corneas of Sesn $2^{-l-}$ mice with $\mathrm{N}$-acetylcysteine (NAC), an inhibitor of ROS, to evaluate whether ROS promote corneal wound healing. Wound closure of the NAC-treated corneas was delayed as compared to that of DMSO-treated corneas after $24 \mathrm{~h}$ (Fig. 5b, c). Moreover, treatment with NAC inhibited wound closure in hCET cells expressing Sesn2 shRNA, whereas DMSOtreated cells were completely healed after $24 \mathrm{~h}$ (Fig. 5d, e).
These data suggest that ROS promote corneal wound healing. To investigate whether ROS regulate YAP activity, the injured corneal epithelial cells of Sesn $2^{-1-}$ mice were treated with NAC or DMSO and analyzed by immunostaining with an anti-YAP antibody. YAP protein was detected predominantly in the nucleus of DMSOtreated corneal epithelial cells, whereas it was expressed in the cytoplasm of NAC-treated cells (Fig. 6a). Fluorescence intensity of the nuclear YAP in NAC-treated corneal 
a

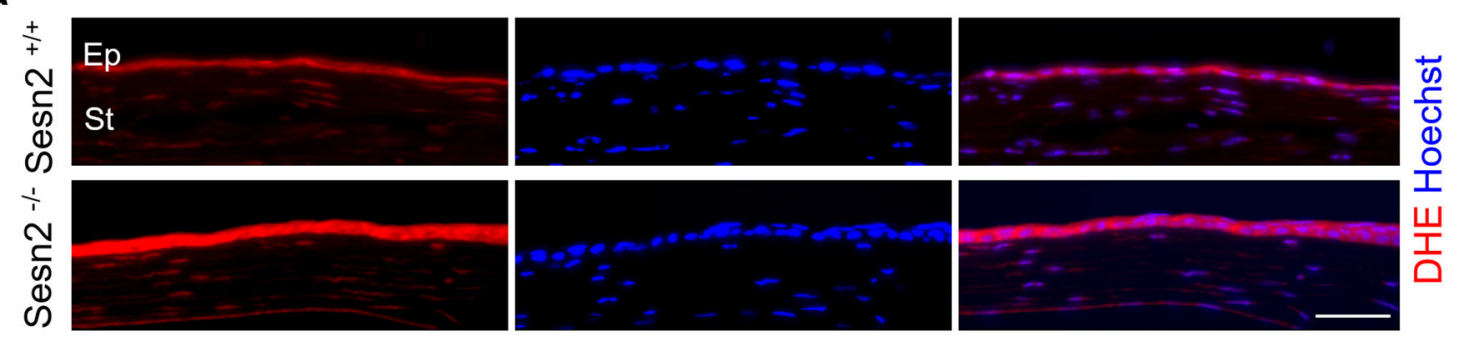

b

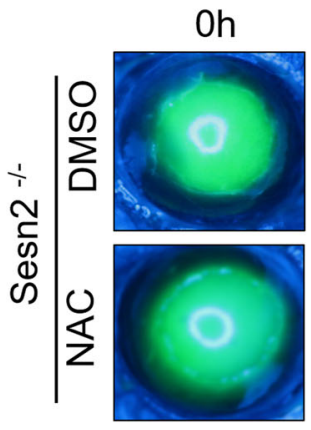

d

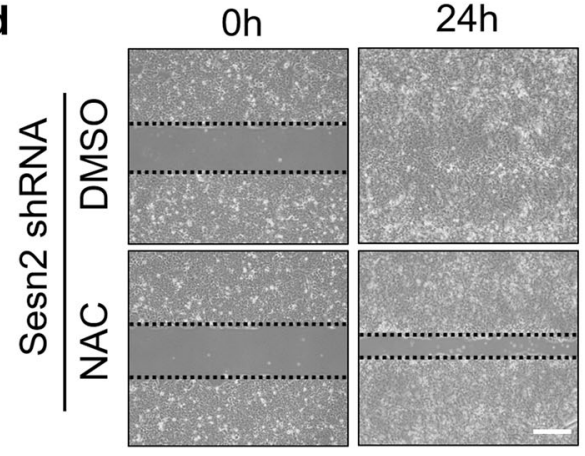

$24 \mathrm{~h}$

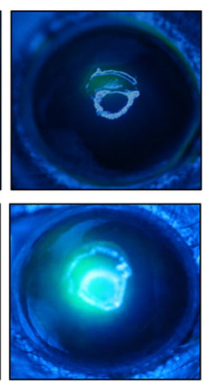

e

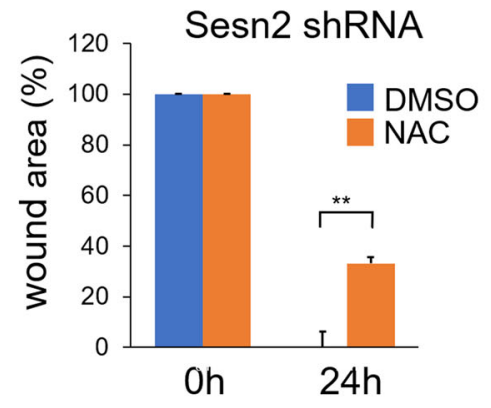

Fig. 5 ROS levels increase in Sesn2-deficient corneal epithelium, and the inhibition of ROS suppresses corneal wound healing. a Detection of ROS by DHE staining in the sections of the injured corneas from Sesn $2^{-/-}$and Sesn2 $2^{+/+}$mice. DHE fluorescence intensity in the corneas of Sesn $2^{-/-}$ mice was significantly higher than that in the corneas of Sesn $2^{+/+}$mice. $\mathbf{b}$ In vivo corneal epithelial wound healing of Sesn $2^{-/-}$mice after injury using a $2 \mathrm{~mm}$ punch. Each cornea was treated with $2 \mathrm{mM}$ NAC or DMSO after injury. c Quantitative analysis of the corneal wound area of Sesn2 ${ }^{-1-}$ mice treated with NAC or DMSO. Inhibition of ROS suppressed corneal wound healing. $\mathbf{d}$ In vitro wound healing assay of hCET cells expressing Sesn2 shRNA treated with 2 mM NAC or DMSO. e Wound closure in NAC-treated hCET cells was delayed than that in DMSO-treated cells. Error bars represent the means \pm SD of three independent experiments. Two-tailed Student's $t$-test $\left.{ }^{* *} p<0.01\right)$. Scale bar, $50 \mu \mathrm{m}$ in $(\mathbf{a}) ; 300 \mu \mathrm{m}$ in $(\mathbf{d})$.

epithelial cells was significantly lower than that in DMSOtreated cells (Fig. 6b). These results indicate that ROS production induces YAP nuclear accumulation. To determine whether ROS regulate YAP activity, we treated hCET cells with $\mathrm{H}_{2} \mathrm{O}_{2}$ and performed western blotting using an anti-phospho-YAP antibody. $\mathrm{H}_{2} \mathrm{O}_{2}$ treatment decreased YAP phosphorylation compared to control (Fig. 6c). The expression level of phospho-YAP protein in hCET cells treated with both $\mathrm{H}_{2} \mathrm{O}_{2}$ and NAC was higher than in hCET cells treated with only $\mathrm{H}_{2} \mathrm{O}_{2}$ (Fig. 6d). Taken together, these results suggest that Sesn2 deficiency increases ROS production and activates YAP in corneal epithelial cells.

\section{Discussion}

Corneal wound healing is essential for the maintenance of corneal transparency and normal visual acuity. Corneal epithelial cell proliferation is a key event in corneal wound healing. However, the molecular mechanisms underlying corneal epithelial cell proliferation are unclear. Here, we identified Sesn2 as a negative regulator of the proliferation of basal epithelial cells in the corneal wound healing process. We also revealed a novel unreported mechanism whereby Sesn2 suppresses YAP activity in an AMPKindependent manner.

Previous studies have demonstrated that Sesn2 suppresses the proliferation of human carcinoma cells, 

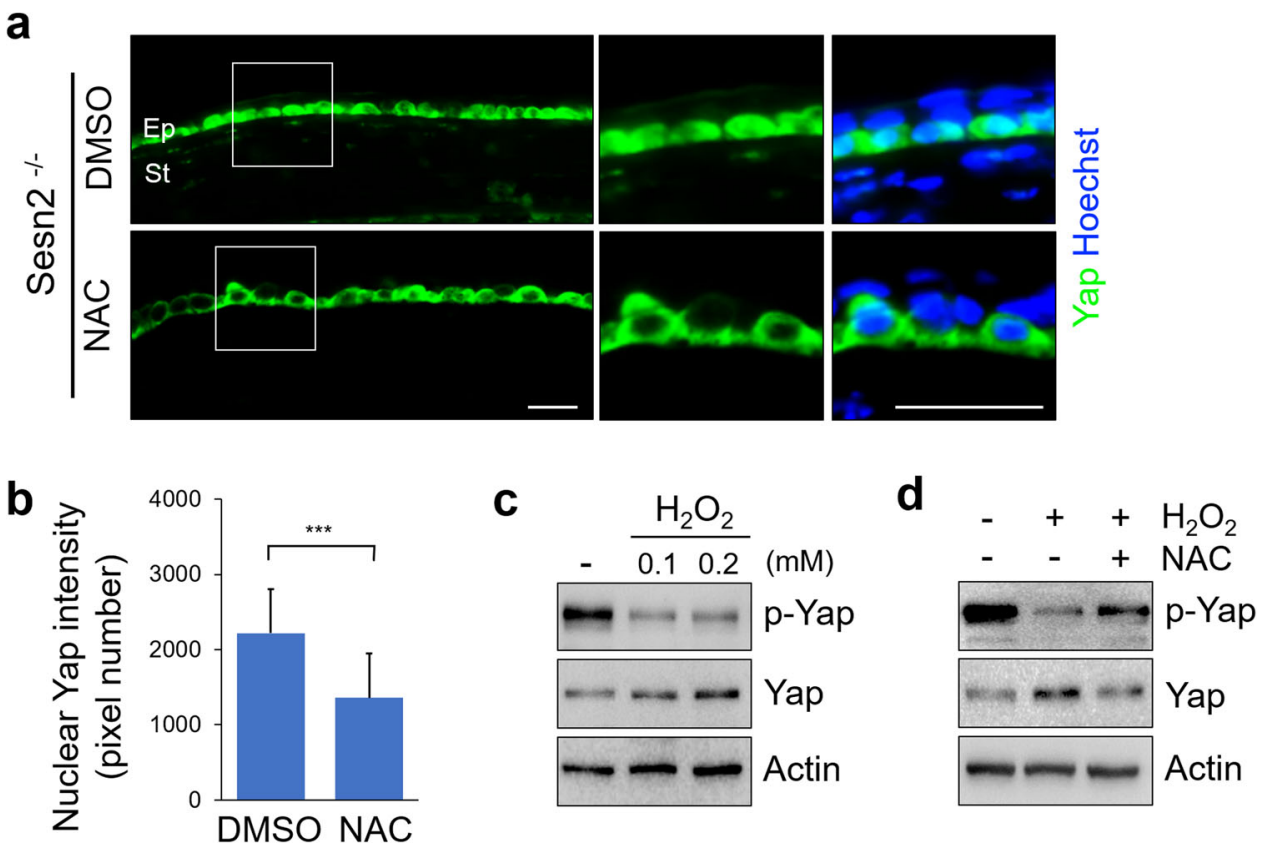

Fig. 6 YAP is activated by increased ROS production. a Sections of injured corneas from Sesn ${ }^{-1-}$ mice treated with 2 mM NAC or DMSO were immunostained using an anti-YAP antibody. Treatment with NAC decreased YAP nuclear localization. $\mathbf{b}$ Fluorescence intensity of nuclear YAP in corneal epithelial cells treated with NAC or DMSO. The nuclear YAP fluorescence intensity of NAC-treated corneal epithelial cells was significantly lower than that of DMSO-treated cells. c Treatment with $\mathrm{H}_{2} \mathrm{O}_{2}$ increased YAP activity. hCET cells were treated with 0.1 or $0.2 \mathrm{mM} \mathrm{H} \mathrm{H}_{2}$ for $2 \mathrm{~h}$. Cell lysates were subjected to western blotting with phospho-YAP, YAP, and actin antibodies. $\mathbf{d}$ Treatment with NAC suppressed YAP activity. hCET cells were treated with $0.2 \mathrm{mM} \mathrm{H}_{2} \mathrm{O}_{2}$ or $2 \mathrm{mM} \mathrm{NAC}$ and subjected to western blotting with phospho-YAP, YAP, and actin antibodies. Error bars represent the means $\pm S D$ of three independent experiments. Two-tailed Student's $t$-test $\left({ }^{* * *} p<0.001\right)$. Scale bar, $50 \mu \mathrm{m}$.

fibroblasts, and epithelial cells ${ }^{6,29,30}$. Herein, we found that Sesn2 is downregulated in epithelial cells during corneal wound healing and that corneal wound healing is faster in Sesn $2^{-/-}$mice than in Sesn $2^{+/+}$mice. Moreover, Sesn2 deficiency in corneal epithelial cells promotes their proliferation. Sesn2 suppresses the mTORC1 pathway, which promotes cell growth and proliferation $^{31-33}$. Sesn2 directly interacts with AMPK, which phosphorylates tuberous sclerosis complex 2 (TSC2) and Raptor and inhibits the mTORC1 signaling pathway ${ }^{6,34,35}$. We showed that phosphorylated S6 was upregulated in Sesn2-deficient corneal epithelial cells in vitro and in vivo. Re-expression of Sesn2 decreased the phosphorylation of S6 protein (Supplementary Fig. S3a). We also found that the inhibition of mTORC1 activity by rapamycin delayed wound closure in the corneal epithelium of Sesn ${ }^{+/+}$and Sesn $2^{-1-}$ mice. These results suggest that Sesn2 acts as a negative regulator of proliferation by inhibiting the mTORC1 signaling pathway in corneal wound healing.

YAP plays an important role in epithelial cell proliferation $^{36-39}$. Upon dephosphorylation at the serine 127 site, YAP is translocated into the nucleus and is subsequently activated to promote the transcription of genes associated with proliferation ${ }^{23,40}$. Our results showed that YAP nuclear localization is increased in basal epithelial cells during corneal wound healing and that the number of these cells increased to a greater extent in Sesn $2^{-1-}$ mice. Treatment with verteporfin, an inhibitor of YAP, resulted in delayed corneal epithelial wound healing in Sesn $2^{-1-}$ mice. Moreover, YAP knockdown inhibited the proliferation of Sesn2-deficient hCET cells. These results suggest that YAP activation is required to regulate cell proliferation by Sesn2. A recent study showed that YAP is inactivated upon phosphorylation by $\mathrm{AMPK}^{41}$. This result indicates that Sesn2 can potentially regulate YAP activity through AMPK. However, YAP activity was not affected when Sesn2-deficient hCET cells were treated with the AMPK activator AICAR and mTORC1 inhibitor rapamycin, although mTORC1 activity was decreased in these cells. Sesn 2 can suppress ROS production through its antioxidant activity ${ }^{42}$. We observed that ROS levels in the corneal epithelium of $\operatorname{Sesn} 2^{-/-}$mice were higher than those in $\operatorname{Sesn} 2^{+/+}$mice during corneal wound healing. As signaling molecules, ROS are involved in multiple intrinsic and extrinsic signaling pathways to stimulate cell proliferation ${ }^{43,44}$. A previous study showed that ROS are essential for EGFstimulated corneal epithelial cell proliferation and wound healing ${ }^{45}$. Our results suggest that ROS can activate YAP 
to promote proliferation. The expression of phosphorylated YAP protein was decreased upon treatment with $\mathrm{H}_{2} \mathrm{O}_{2}$ to induce ROS production. However, treatment with NAC rescued the expression of phosphorylated YAP protein. Moreover, the nuclear localization of YAP in NAC-treated corneal epithelial cells was decreased with delayed wound closure. Oxidative stress can modulate YAP activity ${ }^{46} \cdot \mathrm{H}_{2} \mathrm{O}_{2}$ stimulation induces the binding of TRAF2 to MST1, leading to MST1 activation ${ }^{47}$. MST1/2 are also activated and promote cell death in response to oxidative stress ${ }^{48,49}$. However, YAP forms a complex with FOXO1 and activates FOXO1-mediated transcription of antioxidant genes and subsequently reduces oxidative stress. This process implicates a functional role for YAP in ROS scavenging ${ }^{50}$. Interestingly, a recent study showed increased expression of YAP protein and cell proliferation in neural progenitor cells treated with $\mathrm{H}_{2} \mathrm{O}_{2}{ }^{51}$. Another study also reported that elevated intracellular ROS increase YAP protein expression and decrease YAP phosphorylation but does not affect MST1/2 or LATS1 ${ }^{52}$. Therefore, we speculate that ROS mediate YAP activation independent of the canonical Hippo pathway in Sesn2deficient corneal epithelial cells, although the precise mechanism is not clear. In conclusion, our findings indicate that the downregulation of Sesn2 induces YAP activation through ROS production in corneal wound healing, while activation of mTORC1 signaling is mediated by AMPK.

Interestingly, the nuclear localization of YAP was predominantly restricted to the central region undergoing reepithelialization during wound healing. In contrast, mTORC1 signaling was activated in both the central and peripheral regions. These results suggest that the downregulation of Sesn2 results in the synergistic activation of YAP and mTORC1 signaling to promote reepithelialization of the corneal wound. A recent study has shown that the expression of YAP in both the cytoplasm and nuclei of basal epithelial cells in the central region and in the peripheral region with epithelial progenitor cells plays a critical role in the maintenance of corneal epithelial progenitor cells ${ }^{27}$. The proliferative potential of corneal epithelial progenitor cells is essential for complete wound repair ${ }^{1}$. Although the precise mechanism regulating YAP activity in corneal epithelial progenitor cells requires further investigation, our findings reveal a novel mechanism for Sesn2-dependent proliferation of corneal epithelial cells involving the functional crosstalk between YAP and the mTOR signaling pathway and suggest that Sesn 2 can serve as a potential therapeutic target for re-epithelialization in wound healing.

\section{Acknowledgements}

This research was supported by NRF grant (NRF-2015R1D1A1A01059277) and KHIDI grant (HI17C2412).

\section{Author details}

'Myung-Gok Eye Research Institute, Konyang University, 158 Gwanjeodong-ro, Seo-gu, Daejeon 35365, South Korea. ${ }^{2}$ Department of Medical Science, Konyang University, 158 Gwanjeodong-ro, Seo-gu, Daejeon 35365, South Korea. ${ }^{3}$ Department of Cell Biology, Konyang University, 158 Gwanjeodong-ro, Seo-gu, Daejeon 35365, South Korea. ${ }^{4}$ Department of Ophthalmology, Dankook University Hospital, Dankook University College of Medicine, Dandaero, Dongnam-gu, Cheonan-si, Chungnam 31116, South Korea

\section{Conflict of interest}

The authors declare that they have no conflict of interest.

\section{Publisher's note}

Springer Nature remains neutral with regard to jurisdictional claims in published maps and institutional affiliations.

Supplementary information accompanies this paper at https://doi.org/ 10.1038/s12276-020-0446-5.

Received: 29 November 2019 Accepted: 8 April 2020. Published online: 12 June 2020

\section{References}

1. Tseng, S. C. G. Concept and application of limbal stem cells. Eye 3, 141-157 (1989).

2. Sun, T. T. \& Lavker, R. M. Corneal epithelial stem cells: past, present, and future J. Investig. Dermatol. Symp. Proc. 9, 202-207 (2004).

3. Ljubimov, A. V. \& Saghizadeh, M. Progress in corneal wound healing. Prog. Retin. Eye Res. 49, 17-45 (2015).

4. Lee, J. H., Budanov, A. V. \& Karin, M. Sestrins orchestrate cellular metabolism to attenuate aging. Cell Metab. 18, 792-801 (2013).

5. Budanov, A. V., Sablina, A. A., Feinstein, E., Koonin, E. V. \& Chumakov, P. M. Regeneration of peroxiredoxins by p53-regulated Sestrins, homologs of bacterial AhpD. Science 304, 596-600 (2004).

6. Budanov, A. V. \& Karin, M. p53 target genes Sestrin1 and Sestrin2 connect genotoxic stress and mTOR signaling. Cell 134, 451-460 (2008).

7. Ho, A., Cho, C. S., Namkoong, S., Cho, U. S. \& Lee, J. H. Biochemical basis of Sestrin physiological activities. Trends Biochem. Sci. 41, 621-632 (2016).

8. Zoncu, R., Efeyan, A. \& Sabatini, D. M. mTOR: from growth signal integration to cancer, diabetes and ageing. Nat. Rev. Mol. Cell Biol. 12, 21-35 (2011).

9. Laplante, M. \& Sabatini, D. M. mTOR signaling in growth control and disease. Cell 149, 274-293 (2012).

10. Ruvinsky, I. et al. Ribosomal protein $\mathrm{S} 6$ phosphorylation is a determinant of cell size and glucose homeostasis. Genes Dev. 19, 2199-2211 (2005).

11. Clohessy, J. G., Reschke, M. \& Pandolfi, P. P. Found in translation of mTOR signaling. Cell Res. 22, 1315-1318 (2012).

12. Shin, Y. J. et al. Chemical injury-induced corneal opacity and neovascularization reduced by rapamycin via TGF- $\beta 1 /$ ERK pathways regulation. Invest. Ophthalmol. Vis. Sci. 54, 4452-4458 (2013).

13. Lee, K. S. et al. Bevacizumab and rapamycin can decrease corneal opacity and apoptotic keratocyte number following photorefractive keratectomy. Invest. Ophthalmol. Vis. Sci. 53, 7645-7653 (2012).

14. Shi, W., Gao, H., Xie, L. \& Wang, S. Sustained intraocular rapamycin delivery effectively prevents high-risk corneal allograft rejection and neovascularization in rabbits. Invest. Ophthalmol. Vis. Sci. 47, 3339-3344 (2006).

15. Zapata, G., Racca, L., Tau, J. \& Berra, A. Topical use of rapamycin in herpetic stromal keratitis. Ocul. Immunol. Inflamm. 20, 354-359 (2012).

16. Halder, G. \& Johnson, R. L. Hippo signaling: growth control and beyond. Development 138, 9-22 (2011).

17. Pan, D. The Hippo signaling pathway in development and cancer. Dev. Cell. 19, 491-505 (2010).

18. Zhao, B., Lei, Q. Y. \& Guan, K. L. The Hippo-YAP pathway: new connections between regulation of organ size and cancer. Curr. Opin. Cell Biol. 20, 638-646 (2008).

19. Zhou, D. et al. Mst1 and Mst2 protein kinases restrain intestinal stem cell proliferation and colonic tumorigenesis by inhibition of Yes-associated protein (Yap) overabundance. Proc. Natl Acad. Sci. USA 108, E1312-E1320 (2011). 
20. Zhao, B., Li, L., Lei, Q. \& Guan, K. L. The Hippo-YAP pathway in organ size control and tumorigenesis: an updated version. Genes Dev. 24, 862-874 (2010).

21. Lei, Q. Y. et al. TAZ promotes cell proliferation and epithelial-mesenchymal transition and is inhibited by the Hippo pathway. Mol. Cell Biol. 28, 2426-2436 (2008).

22. Zhao, B. et al. Inactivation of YAP oncoprotein by the Hippo pathway is involved in cell contact inhibition and tissue growth control. Genes Dev. 21, 2747-2761 (2007).

23. Dong, J. et al. Elucidation of a universal size-control mechanism in Drosophila and mammals. Cell 130, 1120-1133 (2007).

24. Huang, J., Wu, S., Barrera, J., Matthews, K. \& Pan, D. The Hippo signaling pathway coordinately regulates cell proliferation and apoptosis by inactivating Yorkie, the Drosophila homolog of YAP. Cell 122, 421-434 (2005).

25. Hong, W. \& Guan, K. L. The YAP and TAZ transcription co-activators: key downstream effectors of the mammalian Hippo pathway. Semin. Cell Dev. Biol. S23, 785-793 (2012)

26. Hao, Y., Chun, A., Cheung, K., Rashidi, B. \& Yang, X. Tumor suppressor LATS1 is a negative regulator of oncogene YAP. J. Biol. Chem. 283, 5496-5509 (2008).

27. Kasetti, R. B. et al. Study of corneal epithelial progenitor origin and the Yap1 requirement using keratin 12 lineage tracing transgenic mice. Sci. Rep. 6 , 35202 (2016).

28. Liu-Chittenden, Y. et al. Genetic and pharmacological disruption of the TEADYAP complex suppresses the oncogenic activity of YAP. Genes Dev. 26, 1300-1305 (2012).

29. Budanov, A. V. et al. Identification of a novel stress-responsive gene Hi95 involved in regulation of cell viability. Oncogene 21, 6017-6031 (2002).

30. Luo, C. et al. SESN2 negatively regulates cell proliferation and casein synthesis by inhibition the amino acid-mediated mTORC1 pathway in cow mammary epithelial cells. Sci. Rep. 8, 3912 (2018).

31. Wei, J. L. et al. Sestrin 2 suppresses cells proliferation through AMPK/mTORC1 pathway activation in colorectal cancer. Oncotarget 8, 49318-49328 (2017).

32. Fingar, D. C. et al. mTOR controls cell cycle progression through its cell growth effectors S6K1 and 4E-BP1/eukaryotic translation initiation factor 4E. Mol. Cell Biol. 24, 200-216 (2004).

33. Wullschleger, S., Loewith, R. \& Hall, M. N. TOR signaling in growth and metabolism. Cell 124, 471-484 (2006).

34. Hardie, D. G., Ross, F. A. \& Hawley, S. A. AMPK: a nutrient and energy sensor that maintains energy homeostasis. Nat. Rev. Mol. Cell Biol. 13, 251-262 (2012).

35. Ben-Sahra, I. et al. Sestrin2 integrates Akt and mTOR signaling to protect cells against energetic stress-induced death. Cell Death Differ. 20, 611-619 (2013).

36. Zhao, B., Tumaneng, K. \& Guan, K.-L. The Hippo pathway in organ size control, tissue regeneration and stem cell self-renewal. Nat. Cell Biol. 13, 877-883 (2011).
37. Ramos, A. \& Camargo, F. D. The Hippo signaling pathway and stem cell biology. Trends Cell Biol. 22, 339-346 (2012).

38. Pan, D. Hippo signaling in organ size control. Genes Dev. 21, 886-897 (2007).

39. Barry, E. R. \& Camargo, F. D. The Hippo superhighway: signaling crossroads converging on the Hippo/Yap pathway in stem cells and development. Curr. Opin. Cell Biol. 25, 247-253 (2013).

40. Wang, K., Degerny, C., Xu, M. \& Yang, X. J. YAP, TAZ, and Yorkie: a conserved family of signal-responsive transcriptional coregulators in animal development and human disease.Biochem. Cell Biol. 87, 77-91 (2009).

41. DeRan, $M$. et al. Energy stress regulates Hippo-YAP signaling involving AMPK-mediated regulation of angiomotin-like 1 protein. Cell Rep. 9, 495-503 (2014).

42. Rhee, S. G. \& Bae, S. H. The antioxidant function of sestrins is mediated by promotion of autophagic degradation of Keap1 and Nrf2 activation and by inhibition of mTORC1. Free Radic. Biol. Med. 88, 205-211 (2015).

43. Hole, P. S. et al. Ras-induced reactive oxygen species promote growth factorindependent proliferation in human CD34+ hematopoietic progenitor cells. Blood 115, 1238-1246 (2010).

44. Chowdhury, R., Chatterjee, R., Giri, A. K., Mandal, C. \& Chaudhuri, K. Arsenicinduced cell proliferation is associated with enhanced ROS generation, Erk signaling and CyclinA expression. Toxicol. Lett. 198, 263-271 (2010).

45. Huo, Y. et al. Reactive oxygen species (ROS) are essential mediators in epidermal growth factor (EGF)-stimulated corneal epithelial cell proliferation, adhesion, migration, and wound healing. Exp. Eye Res. 89, 876-886 (2009).

46. Meng, Z., Moroishi, T. \& Guan, K.-L. Mechnisms of Hippo pathway regulation. Genes Dev. 30, 1-17 (2016).

47. Roh, K.-H. \& Choi, E.-J. TRAF2 functions as an activator switch in the reactive oxygen species-induced stimulation of MST1. Free Radic. Biol. Med 91, 105-113 (2016).

48. Geng, J. et al. Kinases Mst1 and Mst2 positively regulate phagocytic induction of reactive oxygen species and bactericidal activity. Nat. Immunol. 16, 1142-1152 (2015).

49. Lehtinen, M. K. et al. A Conserved MST-FOXO Signaling Pathway Mediates Oxidative-Stress Responses and Extends Life Span. Cell 125, 987-1001 (2006).

50. Shao, D. et al. A functional interaction between Hippo-YAP signalling and FoxO1 mediates the oxidative stress response. Nat. Commun. 5, 3315 (2014).

51. Ji, F., Shen, T., Zou, W. \& Jiao, J. UCP2 regulates embryonic neurogenesis via ROS-mediated Yap alternation in the developing neocortex. Stem Cells 35 , 1479-1492 (2017).

52. Dixit, D., Ghildiyal, R., Anto, N. P. \& Sen, E. Chaetocin-induced ROS-mediated apoptosis involves ATM-YAP1 axis and JNK-dependent inhibition of glucose metabolism. Cell Death Dis. 5, e1212-e1212 (2014). 